

$-2=-\cdots$

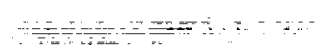
$=-1=-7$

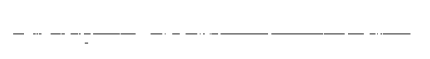

$(-\cdots \cdot-\cdots$

$\div:-=-$

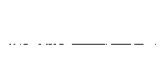

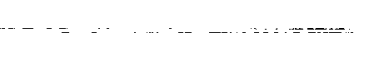

- $\quad-\quad-\cdots-\cdots$

$-\quad-\cdots-\cdots$

- .

- - - - - - -

-

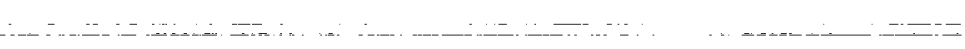

a

$\ldots-n==\quad \ldots \ldots$

$=-1-\cdots+\cdots+\cdots+\cdots$

$-\cdots-\cdots$ 


\title{
COMPUTATIONAL SIMULATION OF MATRIX MICROSLIP BANDS IN SiC/Ti-15 COMPOSITE
}

\author{
S.K. Mital \\ University of Toledo \\ Toledo, Ohio 43606 \\ and \\ H.-J. Lee, P.L.N. Murthy, and C.C. Chamis \\ National Aeronautics and Space Administration \\ Lewis Research Center \\ Cleveland, Ohio 44135
}

\section{ABSTRACT}

Computational simulation procedures are used to identify the key deformation mechanisms for a $[0]_{8}$ and a $[90]_{8} \mathrm{SiC} / \mathrm{Ti}-15$ metal matrix composite. The computational simulation procedures employed consist of a three-dimensional finite-element analysis and a micromechanics based computer code METCAN. The interphase properties used in the analysis have been calibrated using the METCAN computer code with the $[90]_{8}$ experimental stress-strain curve. Results of the simulation show that although shear stresses are sufficiently high to cause the formation of some slip bands in the matrix concentrated mostly near the fibers, the nonlinearity in the composite stress-strain curve in the case of the $[90]_{8}$ composite is dominated by interfacial damage, such as microcracks and debonding rather than microplasticity. The stress-strain curve for the $[0]_{8}$ composite is largely controlled by the fibers and shows only slight nonlinearity at higher strain levels that could be the result of matrix microplasticity.

\section{INTRODUCTION}

High temperature metal matrix composites (HTMMC) are potential structural materials for advanced propulsion systems demanding high operational temperatures $\left(400\right.$ to $1100^{\circ} \mathrm{C}$ ). High stiffness to weight ratios, tailorable properties, dimensional stability, and hygral (moisture) resistance make these materials especially attractive for use in aerothermal structural applications. However, before HTMMC can be used in these critical applications, a thorough identification and understanding of their deformation behavior is required.

Recently, matrix plasticity in the form of slip bands in $\mathrm{SiC}$ / Ti-15 has been observed experimentally by Lerch (1991) using a post-heat treatment and etching method. These slip bands were observed in specimens strained to the nonlinear portions of their stress-strain curves but prior to failure. Results for $[0]_{8}$ and $[90]_{8}$ specimens with the fiber volume ratio of 0.34 show that slip bands are concentrated mostly around the fibers and some interfacial debonding is observed for the $[90]_{8}$ specimen. However, no definite conclusions are made regarding the hierarchy of deformation. Similar studies conducted on $\mathrm{SiC} / \mathrm{Ti}-15$ by Newaz and Majumdar (1991) led them to conclude that plasticity is the dominant mechanism for the $[0]_{8}$ specimen. On the other hand, the $[90]_{8}$ specimen is controlled by both damage and plasticity. Newaz and Majumdar also suggest that the Poisson's ratio can provide additional information regarding the amount of plasticity and damage that occurs in SiC/Ti-15. They suggest that an increase in major Poisson's ratio is indicative of plastic deformation while a decrease corresponds to the dominance of damage in the composite.

The objective of this paper is to computationally simulate the stress-strain behavior of both $[0]_{8}$ and $[90]_{8} \mathrm{SiC} / \mathrm{Ti}-15 \mathrm{com}-$ posites with a fiber volume ratio of 0.34 using METCAN and three-dimensional finite-element analysis (FEA). The resulting microstresses from both analyses are evaluated for the possibility of formation of matrix microslip bands and the key deformation mechanisms are identified. The computational simulation procedures consisting of a three-dimensional finite-element analysis and a micromechanics based computer code METCAN, are described briefly in the following sections.

\section{METCAN COMPUTER CODE}

METCAN stands for Metal Matrix Composite Analyzer and is a computer code developed at NASA Lewis Research Center to simulate the high temperature nonlinear behavior of metal matrix composites. METCAN treats material nonlinearity at the constituent (fiber, matrix, and interphase) level, where the behavior of the material is modeled using the dependence of a constituent's properties in a time-temperature-stress "material behavior space." The composite properties are synthesized from the constituent instantaneous properties by virtue of composite micromechanics, composite macromechanics, and laminate theory. Factors which affect the

"NASA Resident Research Associate at Lewis Research Center. 
behavior of the composite properties include the fabrication process variables, in-situ fiber and matrix properties, and/or the properties of an interphase between the fiber and matrix. A unique aspect of the METCAN code is an integrated cyclic arrangement which defines the computational effort for each load increment as shuwn in Fig. 1. Another feature is the use of a "multifactor interaction relationship" (MFIR) to represent the various nonlinearities and their interactions in the constituents. The multifactor interaction relationship (MFIR) and the reasons for its selection are shown in Fig. 2. More information regarding METCAN and the efforts to verify the code can be found in Chamis et al. (1990) and Lee et al: (1991).

\section{FINITE EILEMENT MODEL.}

The finite element model used in this simulation consists of a group of nine fibers, all unidirectional, in a three-by-three unit cell array ("nine-cell model") as shown in Fig. 3. Since both the composites examined are unidirectional and to keep the time required in the analysis to a reasonable limit, only three plies (or three-by-three unit cell array) have been simulated in the finiteelement analysis. The fiber diameter is taken as $150 \mu \mathrm{m}$ and the interphase thickness is assumed to be approximately 3 percent of the fiber diameter with a fiber volume ratio of 0.34 . There are 16 elements ("bays") along the length of the fiber. Each unit cell, as shown in Fig. 3, consists of 40 hexahedron (six-sided) solid elements and eight pentahedron (five-sided) solid elements for a total of 6912 elements and 6953 nodes in the model. This corresponds to approximately 20000 degrees of freedom in the model, which provides sufficient detail of the stress distribution in the composite for the purposes of this study. A general purpose finite-element analysis computer code MSCNNASTRAN (1985) has been used to carry out the analysis. For longitudinal loading, uniform boundary displacements are specified in the $1-1$ (along the fiber) direction. For transverse loading, uniform boundary displacements are specified in the 2-2 (transverse to the fiber) direction. The resulting nodal forces to sustain the applied displacements are computed by the finite-element analysis and the resulting longitudinal or transverse stress is obtained by dividing the total force by the appropriate cross-sectional area.

\section{COMPUTATIONAL SIMULATION PROCEDURES}

The computational simulation procedures used in this study begin with the METCAN analysis to determine some of the constituents material properties for the finite-element analysis. Although bulk properties of the fiber and matrix are available (Lerch, 1989) and listed in Table I, additional properties for the interphase are required to conduct an accurate finite-element analysis. However, since experimental values for the interphase properties are unavailable, a calibration is conducted using METCAN with the $[90]_{8}$ experimental stress-strain curve to determine the interphase modulus and strength. Also, previous METCAN verification conducted on SiC/Ti-15 (Chamis et al., 1990) has shown that the

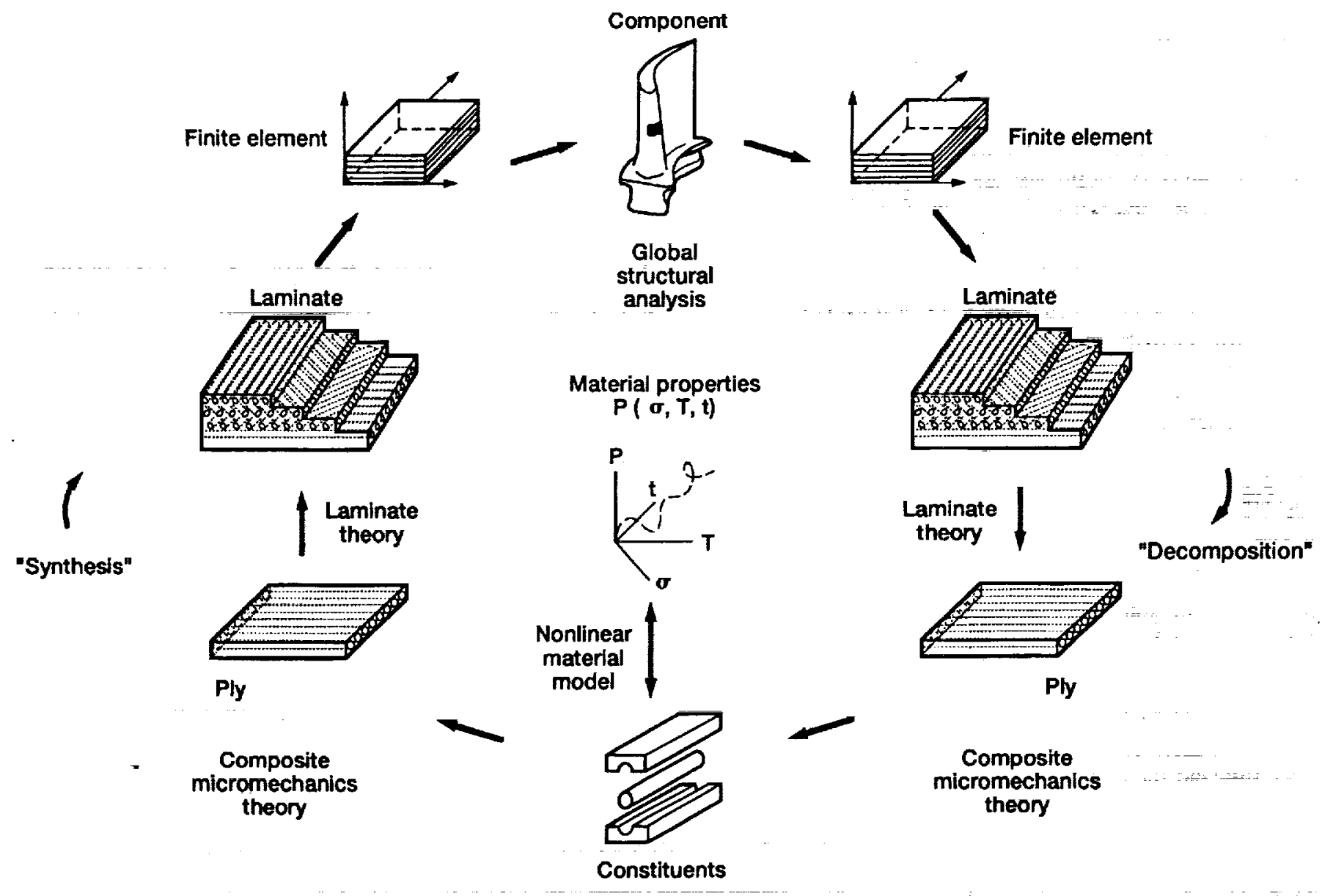

Figure 1.-Integrated approach to metal-matrix composite analysis. 
$\frac{P}{P_{O}}=\left[\frac{T_{F}-T}{T_{F}-T_{O}}\right]^{n}\left[\frac{S_{F}-\sigma}{S_{F}-\sigma_{O}}\right]^{m}\left[\frac{S_{F}-\dot{\sigma}_{O}}{\dot{S}_{F}-\dot{\sigma}_{O}}\right]^{l}\left[\frac{t_{F}-\dot{T}_{\dot{T}_{F}}-\dot{T}_{O}}{{ }^{k}}\right]^{k}\left[\frac{R_{F}-R}{R_{F}-R_{O}}\right]^{P} \cdots$

$$
\ldots\left[\frac{N_{M F}-N_{M}}{N_{M F}-N_{M O}}\right]^{q}\left[\frac{N_{T F}-N_{R}}{N_{T F}-N_{T O}}\right]^{t}\left[\frac{t_{F}-t}{t_{F}-t_{O}}\right]^{3} \ldots
$$
Subreglons of
intralaminar

Rationale:

nonunliormity

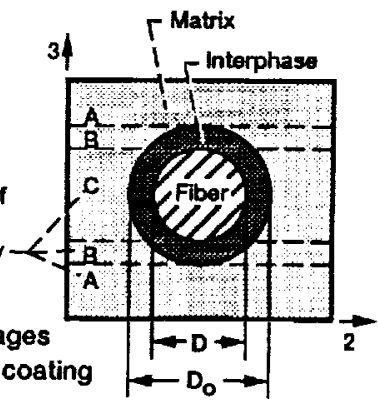

ual effects during most range, rapidly degrading near fing sta

- Representative of the in situ behavior for fiber, matrix, interphase, coating

- Introduction of primitive variables (PV)

- Consistent in situ representation of all constituent properties in terms of PV

- Room-temperature values for reference properties

- Continuous interphase growth

- Simultaneous interaction of all primitive variables

- Adaptability to new materials

- Amenable to verification inclusive of all properties

- Readily adaptable to incremental computational simulation

Notations:

P - property; $T$ - temperature; $\mathbf{S}$ - strength; $\mathrm{R}$ - metallurgical reaction; $\mathrm{N}$ - number of cycles:

$t$ - time; over dot - rate; subscripts: $O$ - reference; $F$ - final; $M$ - mechanical; $T$ - themal

Figure 2.-Assumed multifactor interaction relationship to represent various factors which influence in-situ constituent materials behavior.
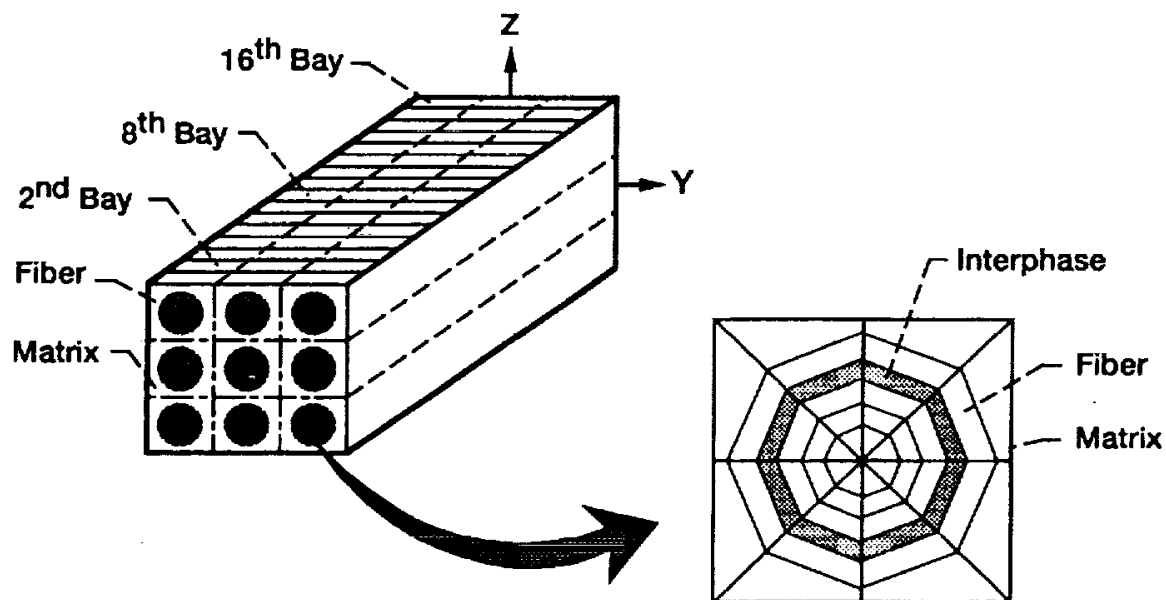

Unit cell

6912 Elements

6953 Nodes

Figure 3. Schematic of model used for finite element analysis. $\mathrm{SiC} / \mathrm{Ti}-15$; fiber volume ratio, 0.34 
Table 1: Constituent Material Properties.

\begin{tabular}{|c|c|c|c|}
\hline & SiC Fiber & Ti-15 matrix & $\begin{array}{c}\text { Interphase } \\
\text { (3\% thickness) }\end{array}$ \\
\hline Density, $\rho, \mathrm{lb} / \mathrm{in} .^{3}$ & 0.110 & 0.172 & 0.172 \\
\hline $\begin{array}{l}\text { Stress free tempera- } \\
\text { ture, } T_{m},{ }^{\circ} \mathrm{F}\end{array}$ & 4870 & 1400 & 1400 \\
\hline Elastic modulus, E, Msi & 62.0 & $\$ 2.3$ & 17.0 \\
\hline Poisson's ratio, $v$ & 0.19 & 0.32 & 0.32 \\
\hline $\begin{array}{l}\text { Coefficient of thermal } \\
\text { expansion, } \alpha, p p m /^{\circ} F\end{array}$ & 2.72 & 4.72 & 4.72 \\
\hline Strength, ksi & 500 & 60 & 48 \\
\hline
\end{tabular}

matrix in the in-situ state will not realize the full bulk matrix strength. Thus, the in-situ matrix strength is also calibrated from the $[90]_{8}$ stress-strain curve.

The calibration procedure consists of three steps. In the first step, the in-situ matrix strength is varied until a match is obtained between the METCAN prediction for strength and the experimentally observed value for the $[90]_{8}$ laminate. The interphase modulus is determined in the second step by matching the initial slope of the METCAN predicted stress-strain curve with the experimental $[90]_{8}$ curve. In the third step, the interphase strength is calibrated by matching the first kink of the experimental $[90]_{8}$ curve. It should be noted that the calibration procedure is performed using only data from the $[90]_{8}$ case. Once the calibrated properties are obtained, they are kept at the same values throughout the study.

After the calibration procedure is completed, three cases are examined using both METCAN and FEA. The first case simulates processing alone. The second and third cases simulate processing followed by a longitudinal loading on a $[0]_{8}$ and a $[90]_{8}$ composite, respectively. Results from each case will be discussed in the following section.

\section{RESULTS AND DISCUSSION}

\section{Processing}

Processing is simulated as a cool down from the processing temperature $\left(700^{\circ} \mathrm{C} / 1292{ }^{\circ} \mathrm{F}\right)$ to room temperature $\left(21^{\circ} \mathrm{C} / 70^{\circ} \mathrm{F}\right)$ by both METCAN and FEA. The METCAN analysis automatically takes into account the nonlinear variation of material properties with temperature and stress via the multifactor interaction relationship mentioned before. The nonlinear material behavior is incorporated into FEA by using material properties generated from the multifactor interaction relationship. For simplicity, the FEA simulation of processing has been carried out in one step using material properties computed at $360.5^{\circ} \mathrm{C} / 6811^{\circ} \mathrm{F}$ (average of 700 and $21^{\circ} \mathrm{C}$ ) by the multifactor interaction relationship using only a temperature dependence. Thus while the METCAN analysis takes into account the variation of material properties with temperature and stress, the constituent material properties depend only on temperature in the finite-element analysis.

The residual stresses that develop in the interphase as a result of processing at approximately the 3 o'clock position (point b) are shown in Fig. 4. For both radial and hoop stresses, METCAN

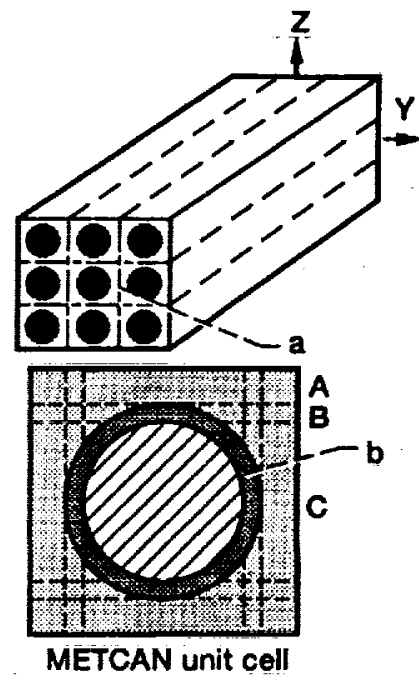

Figure 4.-Results for processing. 
predictions are conservative of FEA. This is expected since METCAN stresses represent average values over a whole region, while FEA determines stress values at a particular point. The large hoop stresses predicted by both analyses ( 35 to $41 \mathrm{ksi}$ ) due to processing alone are sufficiently high to cause the formation of radial microcracks in the interphase. Such radial microcracks have been observed experimentally by MacKay (1990).

\section{$[90]_{8}$ Composite}

The transverse stress-strain curves obtained from METCAN and FEA, along with the experimental curve are shown in Fig. 5. The curves are in good agreement. The furst kink in the stressstrain curve occurs at an applied stress of approximately $42 \mathrm{ksi}$ as interfacial debonding initiates. This applied stress level corresponds to a maximum shear stress of approximately $25 \mathrm{ksi}$. Although this shear stress is large enough to account for the local slip bands observed experimentally, examining the unloading curve in Fig. 6 shows that the nonlinear behavior is dominated by damage. Since there is good agreement in the loading part of the stressstrain curve (Fig. 5), only an average loading curve has been plotted in Fig. 6. The experimental modulus during unloading, as shown in Fig. 6, is significantly reduced in comparison to the loading modulus, which results in a very small permanent set ( 0.0004 total strain). The presence of a permanent set indicates that some plasticity is occurring, but the almost negligible value indicates that a damage mechanism plays a more dominant role. Since experimental observations have indicated both interfacial debonding (Lerch, 1991; Lerch and Saltsman, 1991) and interfacial radial cracks (MacKay, 1990) for this orientation, interfacial debonding is the critical deformation mechanism.

This conclusion is reinforced by the predictions of major Poisson's ratio by METCAN and FEA shown in Fig. 7. METCAN values are shown by the continuous line, while only two points are calculated from FEA. The decrease in Poisson's ratio with increasing strain shown in the figure agrees well with the results observed by Newaz and Majumdar (1991), indicating that the primary deformation mechanism is damage.
$[0]_{8}$ Composite

The longitudinal stress-strain curves at room temperature obtained from METCAN and FEA are shown in Fig. 8, along with the experimental curve. The FEA curve is slightly on the higher (stiffer) side. This may be due to the assumption of only a temperature dependence of the constituent properties for FEA, while METCAN assumes a dependence on temperature and stress. The stress-strain curve is mainly linear with a slight nonlinearity at the higher strain levels. The maximum shear stress obtained from both FEA and METCAN is approximately $30 \mathrm{ksi}$. Once again, although this shear stress is sufficient to cause the formation of matrix slip bands observed experimentally, the mostly linear (elastic) behavior of the $[0]_{8}$ stress-strain curve indicates that plasticity is unlikely to be the dominant deformation mechanism. In order for any slip to take place, some type of damage in the form of either radial microcracks or debonding must exist in the interphase. In other words, the presence of such microslip bands is indicative of interfacial damage, which could arise during fabrication. Previous work by the first author on composite microfracture (Mital et al., 1990) has shown that even an existing interfacial debond will not propagate if the loading is applied along the fiber (longitudinal) direction without a fiber fracture. Therefore, local interphase shear failure appears to be benign under this loading case and the composite behavior is controlled by the fibers. Thus, even with the presence of experimentally observed radial cracks in the interphase (reaction zone cracks) due to processing and the METCAN predicted failure

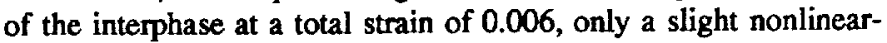
ity is introduced in the stress-strain behavior after the interphase failure. This indicates that the observed matrix plasticity follows reaction zone cracks, and effects the stress-strain curve only at higher strain levels. This conclusion is further supported by the prediction of almost constant major Poisson's ratio for this case as shown in Fig. 9.

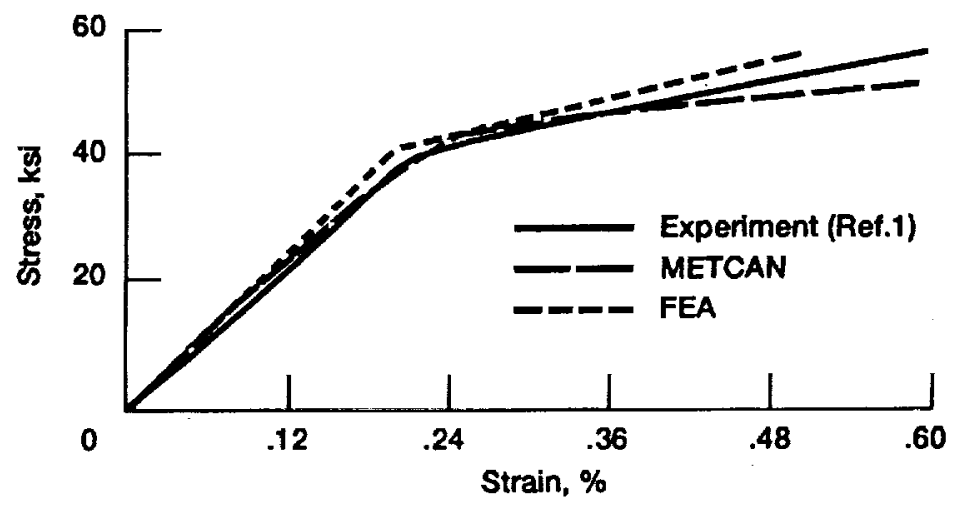

Figure 5.-Room-temperature transverse $[90]_{B}$ stress-strain curve. 


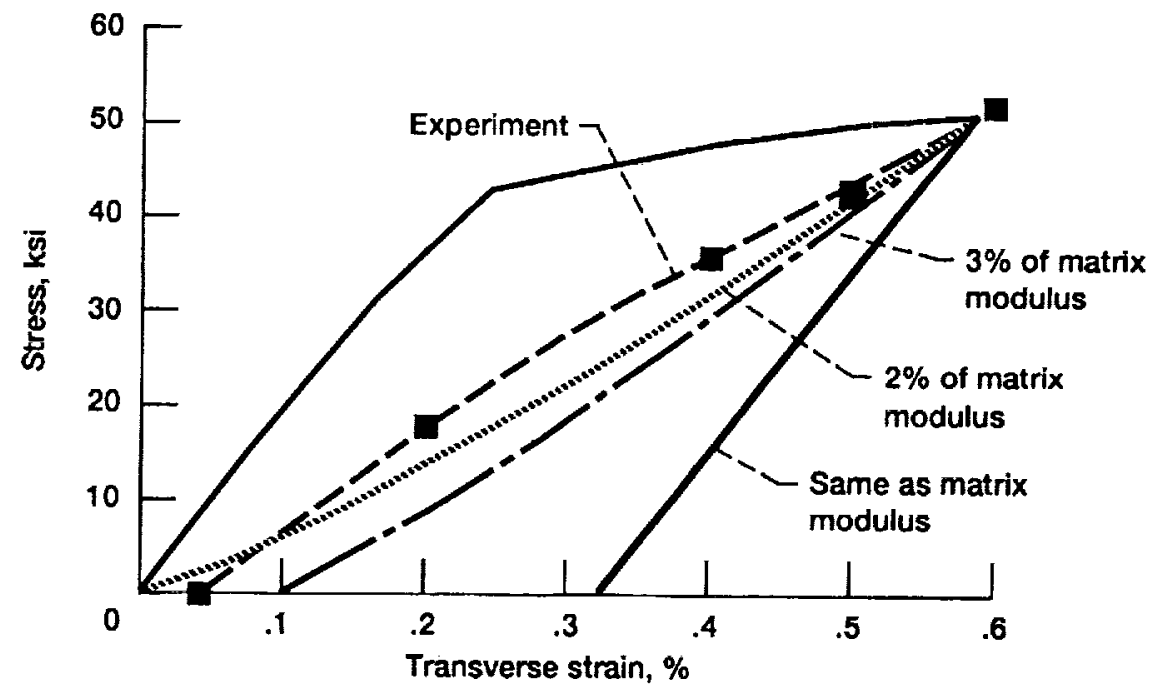

Figure 6. - Transverse $[90]_{8}$ loading/unloading curve.

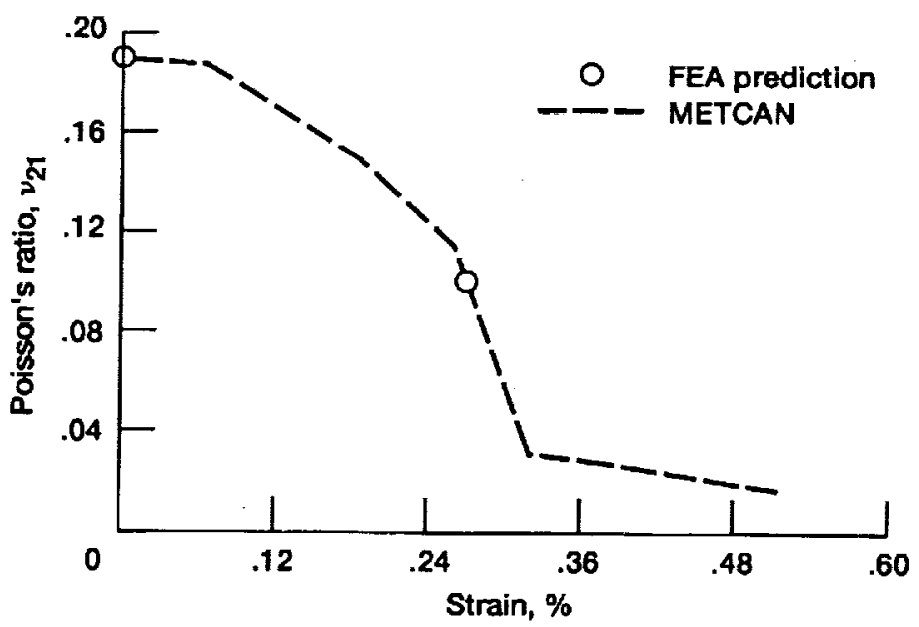

Figure 7.-Variation of $v_{21}$ vs. strain at room temperature.

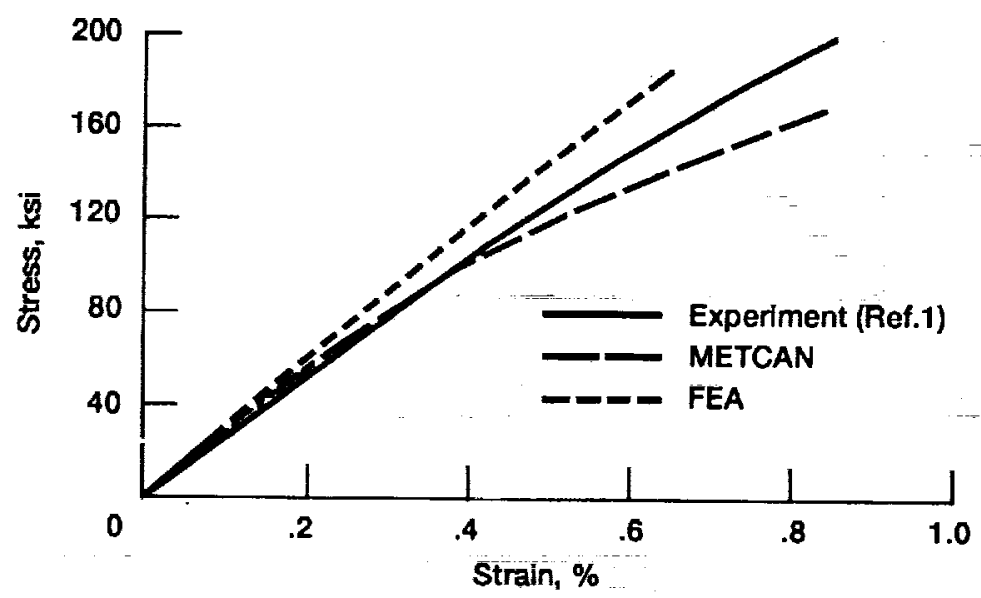

Figure 8.-Room-temperature longitudinal $[0]_{8}$ stress-strain curve. 


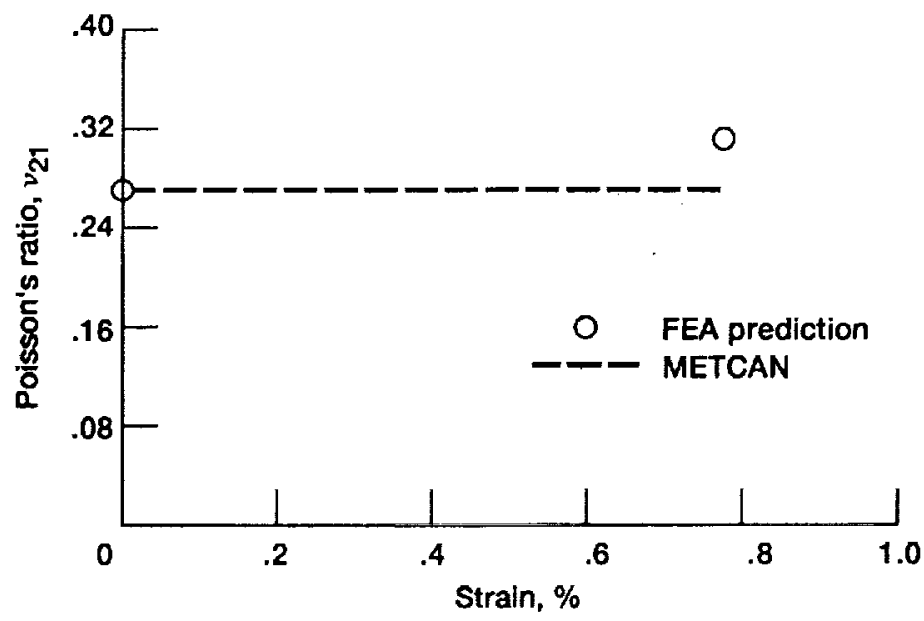

Figure 9.-Variation of $v_{21}$ vs. strain at room temperature.

\section{SUMMARY}

A three-dimensional finite-element analysis and the METCAN computer code were used to computationally simulate the stressstrain behavior of a $[0]_{8}$ and $[90]_{8} \mathrm{SiC} / \mathrm{Ti}-15$ composite. Interphase properties and the in-situ matrix strength were determined by calibrating the METCAN using the experimental results for the $[90]_{8}$ composite. Results indicate that although matrix microslip bands are likely to occur concentrated mostly near the fibers, the nonlinearity in the composite stress-strain curve is dominated by damage either in the form of microcracks or debonding. In the case of the $[90]_{8}$ composite, the unloading modulus is much less than the loading modulus and the permanent set is extremely small, indicating the dominance of damage rather than microplasticity as the key deformation mechanism. In the case of the $[0]_{8}$ composite, the stress-strain behavior shows only a slight nonlinearity at higher strain levels that could be the result of matrix microplasticity. The behavior of $[0]_{8}$ composite is controlled by the fibers in general and hence, any local shear failures are benign in this loading case.

\section{REFERENCES}

Chamis, C.C., et al., 1990, "METCAN Verification Status," NASA TM-103119.

Lee, H.-J., Murthy, P.L.N., and Chamis, C.C., 1991, "METCAN Updates for High Temperature Composite Behavior. Simulation/ Verification," NASA TM-103682.
B.A. Lerch, Private communication, 1989, NASA Lewis Research Center, Cleveland, OH.

Lerch, B.A., 1991, "Matrix Plasticity in SiC/Ti-15-3 Composite," NASA TM-103760.

Lerch, B.A., and Saltsman, J.F., 1991, "Tensile Deformation Damage in SiC Reinforced Ti-15V-3Cr-3Al-3Sn," NASA TM-103620,

MSC/NASTRAN, 1985, Version 65, Vols. I and II, Users Manual, The MacNeal-Schwendler Corporation, Los Angeles, CA.

MacKay, R.A., 1990, "Effect of Fiber Spacing on Interfacial Damage in a Metal Matrix Composite," Scripta Metallurgica, Vol. 24, No. 1, pp. 167-172.

Mital, S.K., Caruso, J.J., and Chamis, C.C., 1990, "Metal Matrix Composites Microfracture: Computational Simulation," Computers and Structures, Vol. 37, No. 2, pp. 141-150.

Newaz, G.M., and Majumdar, B.S., 1991, "Deformation and Failure Mechanisms in Metal Matrix Composites," AD-Vol. 22/ AMD-Vol. 122, Failure Mechanisms in High Temperature Composite Materials, ASME, pp. 55-65. 


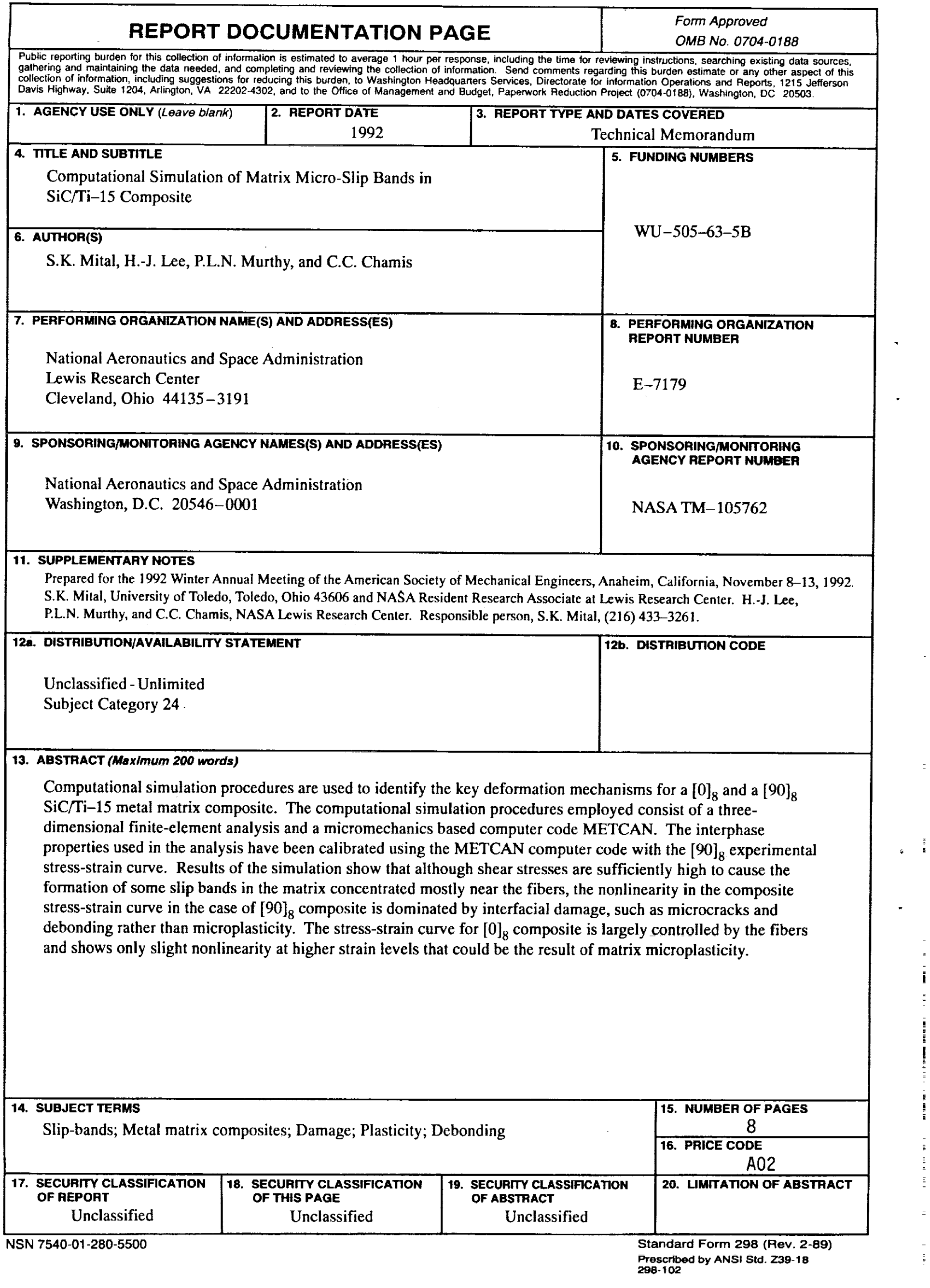

